\title{
BMJ Open Factors influencing the readiness to tackle the burden of ischaemic heart disease in India: a systematic review protocol
}

\author{
Shuvarthi Bhattacharjee (D) , ${ }^{1}$ Nima Yaghmaei (D) , ${ }^{2}$ Cao Tran Le Phuong (D) , \\ Dinesh Neupane (1) ${ }^{4}$
}

To cite: Bhattacharjee S, Yaghmaei N, Phuong CTL, et al. Factors influencing the readiness to tackle the burden of ischaemic heart disease in India: a systematic review protocol. BMJ Open 2021;11:e047464. doi:10.1136/ bmjopen-2020-047464

- Prepublication history and additional supplemental material for this paper are available online. To view these files, please visit the journal online (http://dx.doi.org/10.1136/ bmjopen-2020-047464).

Received 30 November 2020 Accepted 14 July 2021

Check for updates

(C) Author(s) (or their employer(s)) 2021. Re-use permitted under CC BY-NC. No commercial re-use. See rights and permissions. Published by BMJ.

${ }^{1}$ School of Health Sciences, University of Brighton, Brighton, UK

${ }^{2}$ Research \& Development Wing, HumERA Consulting, Toronto, Ontario, Canada

${ }^{3}$ Department of Public Health, University of Medicine and Pharmacy, Ho Chi Minh City, Vietnam

${ }^{4}$ Department of Epidemiology, Johns Hopkins University, Bloomberg School of Public Health, Baltimore, Maryland, USA

Correspondence to Mr Shuvarthi Bhattacharjee; S.Bhattacharjee@brighton.ac.uk

\section{ABSTRACT}

Introduction Ischaemic heart disease (IHD) is one of the leading causes of death and disease burden in India affecting all age groups. To reduce the deaths and tackle the burden of existing IHD, the government approach has been mostly through the National Health Policy (2017) and National Programme for Prevention and Control of Diabetes, Cardiovascular diseases and Stroke. This paper offers a protocol for the systematic review of studies exploring the factors influencing service readiness of the public health system of India to tackle the burden of IHD. Methods and analysis Electronic databases of Embase (Ovid), AMED (Ovid), HMIC (Ovid), BNI (ProQuest), CINAHL (EBSCO), EMCARE (Ovid), PsycINFO (ProQuest), MEDLINE/ PubMed and Web of Science (Clarivate Analytics) will be searched till 2020 for primary studies. Grey literature will be accessed through OpenGrey, TRIP Medical, WHO database, MoHFW website, Open Government Data (OGD) Platform of India and Google Scholar (between 2010 and 2020). Primary studies meeting the eligibility criteria and grey literature published in English between 2010 and 2020 will be included. Data will be analysed through a conceptual framework, and the primary outcome will constitute both quantitative and qualitative data. The quality of included studies will be assessed based on study design. Data will be managed on the COVIDENCE platform. All authors will be involved in data extraction, quality appraisal, data synthesis and formulation of the final draft. Ethics and dissemination This study, being a systematic review, does not involve any clinical trial, primary data collection or empirical study involving humans or animals. Therefore, no ethical permissions were sought by reviewers.

PROSPERO registration number CRD42020219490.

\section{INTRODUCTION}

\section{Rationale}

Ischaemic heart disease (IHD) is the leading cause of deaths and disease burden among adults in India. In 2019, IHD alone claimed approximately 1.5 million deaths in India with a mortality rate of 109.23 deaths per 100000 populations. ${ }^{1}$ Between 2009 and 2019, deaths due to IHD increased significantly by $29.8 \%$

\section{Strengths and limitations of this study}

- The study can inform policymakers, academicians, non-governmental organisations (NGOs) and healthcare administrators with an understanding of the existing gaps in the health system.

- This study can provide options to optimise existing health system resources of India to extend better quality of care for the patients.

- Our use of a deductive approach with a conceptual framework for data analysis may exclude some findings, which might be a limitation.

- India, being a vast country with a decentralised system of health governance, may have varying levels of service readiness across states, making it difficult to generalise study findings for all contexts.

in India and in the year 2019 contributed to $7.79 \%$ of the total disability-adjusted life years (DALYs). IHD affected all age groups, including the country's working population, leading to their premature deaths.

High burden of disease and deaths due to IHD can have significant socioeconomic implications for India. In particular, the working population of India who are earning members within households may suffer premature deaths, leading to loss of individual lives and resulting in economic instability for their dependents. Moreover, healthcare expenditure is majorly met through out-of-pocket (OOP) expenses, presently at $63 \%$ of current health expenditure of India, pushing households towards financial impoverishment. ${ }^{2}$ In such a situation, tackling the disease burden and reducing deaths due to IHD seem necessary to avert loss of individual lives and to prevent possible household financial impoverishment.

In order to reduce the deaths and tackle the burden of IHD in India, the government approach has been mostly through 
context-centric tactics of tackling non-communicable diseases (NCDs) through nationwide health sector policy and programmes. ${ }^{3}$ In agreement with the Global Action Plan for Prevention and Control of NCDs 2013-2020, endorsed by the World Health Assembly in 2013, India has adopted 25 indicators and 9 voluntary goals to reduce $25 \%$ of premature deaths due to NCDs by $2025 .{ }^{45}$ Premature mortality associated with IHD is indicated as unconditional probability of dying due to IHD between the ages of 30 and 70 years. In India, the translation of this global action plan at a national level has occurred through the National Health Policy (NHP) (NHP 2017), and the policy vision is being implemented across states through multiple approaches by the National Programme for Prevention and Control of Cancer, Diabetes, Cardiovascular Diseases and Stroke (NPCDCS). ${ }^{6}{ }^{7}$ Additionally, India's flagship programme on universal health coverage, Ayushman Bharat, comprises the conversion of 150000 primary health centres into health and wellness centres. This measure aims to strengthen primary care services that can prevent and treat NCDs including IHD. ${ }^{8}$ To support the cause, the NHP 2017 planned to raise the general government health expenditure (GGHE) to $2.5 \%$ of the GDP from the usual government allocation of $1.2 \%$ of the GDP in previous years. Moreover, NHP 2017 has suggested NPCDCS to strengthen secondary and tertiary care services, along with primary care NCD interventions, such as 'best buys'.

However, while the plans and commitments have been put in place, the challenges of the existing disease burden of IHD, have overwhelmed public healthcare systems. Additionally, the scarcity of health workers and the poor co-operation between states and the central government in India's decentralised health administrative structure appears to be detrimental towards tackling the disease burden of IHD. ${ }^{10-12}$ Therefore, it becomes necessary to explore the factors influencing readiness of the public health system of India in order to tackle IHD based on the commitments made through the NHP 2017 and other relevant national programmes. Readiness here is defined as the cumulative availability of components required to provide services. $^{13}$

In a nutshell, this is an attempt to explore whether the existing public health system is capable of delivering the government commitments on NHP and national NCD programme to the people of India, through the existing public health system infrastructure, in order to tackle the mortality and morbidity due to IHD.

Our preliminary search on this topic in the Cochrane Database of Systematic Reviews, International Prospective Register of Systematic Reviews (PROSPERO), JBI Evidence Synthesis, CRD/DARE Database and Campbell Collaboration revealed no systematic reviews, scoping reviews, rapid reviews or published protocols related to India concerning readiness of health systems. However, there were reviews focused on health system components for low and middle-income country (LMIC) settings, acute care settings concerning LMICs, monitoring of cardiac patients and risk factors associated with IHD in Asian Indians (included those of Indian origin living outside India), which bear no similarity to our study. ${ }^{14-18}$ Moreover, data from such studies considered LMIC context in a totality which made it difficult to find any specific data for India. The COVID-19 pandemic in India has already witnessed a scarcity of oxygen supply and overwhelmed tertiary care sector. ${ }^{19}$ Further, health system issues like dual practice, poor skill mix and inadequate number of healthcare staff in the public sector have further constrained the existing care system. ${ }^{20}$ In this scenario, people with IHD or at risk of IHD might overstrain the existing care system especially with cases requiring tertiary care services.

\section{Objectives}

The objective of this study is to identify the health system factors that contribute to the facility readiness of the public healthcare system of India in tackling the burden of IHD. To achieve the stated objective, a systematic review to analyse the existing peer-reviewed studies and grey literature is proposed to be undertaken. The analysis will be guided through a conceptual framework, the 'WHO Framework for Action'. ${ }^{21}$

\section{METHODS}

\section{Eligibility criteria}

Population or participants and conditions of interest

Studies involving Indian people living with or at risk of IHD will be included. Primary studies related to such population type published in peer-reviewed journals and grey literature in English published between 2010 and 2020 will be included.

\section{Interventions or exposure}

Relevant primary studies in peer-reviewed journals and grey literature on national programmes of NCD, NHPs (NHP 2012 and NHP 2017), interventions concerned with both prevention and control of IHD in India will be included if published in English between 2010 and 2020.

\section{Comparisons or control groups}

None.

\section{Outcomes of interest}

Data of both quantitative and qualitative nature related to the health system preparedness or readiness to prevent and tackle the burden of IHD in India will be included.

\section{Settings}

Primary, secondary and tertiary care settings within the public health system of India will be included.

\section{Study design}

All forms of study design of both quantitative \& qualitative nature will be included. 
Time frame

2010-2020. This time frame has been chosen considering that the NPCDCS was launched by the Ministry of Health and Family Welfare, Government of India, in $2010 .^{22}$

\section{Reporting characteristics}

Timeline: 2010-2020; Language: English; Publication status: peer reviewed.

\section{Information sources}

Details of information sources are outlined in online supplemental material A: Information Sources \& Representative Search Strategy.

\section{Search strategy}

Electronic databases of EMBASE (Ovid), AMED (Ovid), HMIC (Ovid), BNI (ProQuest), CINAHL (EBSCO), EMCARE (Ovid), PsycINFO (ProQuest), MEDLINE/ PubMed and Web of Science (Clarivate Analytics) will be searched with the use of appropriate Boolean operators (AND, OR \& NOT) until 2020. Appropriate filters matching the reporting characteristics will be used at this stage. All electronic databases (except MEDLINE/ PubMed and Web of Science) will be accessed through the Healthcare Databases for NHS in England (HDAS) of the NHS OpenAthens, UK. HDAS mirrors search results of platforms like Ovid, EBSCO and ProQuest to retrieve results from these databases.

Trial registries like PROSPERO, Cochrane Database of Systematic Reviews, Campbell Collaboration and CRD/ DARE Database will be searched to avoid duplication of existing knowledge on the topic and to identify relevant primary studies from available systematic reviews.

Moreover, citation tracking, footnote chasing, bibliography scan and citation alerts (on Web of Science) will be conducted for a comprehensive search. ${ }^{23} \mathrm{~A}$ combination of free texts and corresponding synonyms of terms comprising but not limited to "Ischemic Heart Disease" AND "Health System" AND "Readiness" AND "India" will be made for the initial search and appropriate preidentified Medical Subject Headings Terms ('Emtree Terms' for Embase) in PubMed/MEDLINE will be included to make a comprehensive search strategy across the electronic databases (online supplemental table 1 added as a representative example). Thesaurus terms appropriate to the databases will be used to account for the variation in such terms across databases.

Grey literature will be accessed through OpenGrey, TRIP Medical, WHO database, MoHFW website, Open Government Data (OGD) Platform of India and Google Scholar (between 2010 and 2020).

Detailed lists of information sources and a representative search result for this study are given in online supplemental material A: Information Sources \& Representative Search Strategy.

\section{STUDY RECORDS}

\section{Data management}

COVIDENCE systematic review software (available at : www.covidence.org) will be employed to manage the retrieved studies and initiate the process of this systematic review. On this software, duplicate articles will be rejected in order to gather a set of unique articles for this study. ${ }^{24}$ All article screening activities, selection of a final set of articles for inclusion in the study after examination of full texts of eligible studies, data extraction and risk of bias assessment will be carried out on COVIDENCE. References will be managed and stored on EndNote. All forms of article selection, data extraction, quality appraisal and risk of bias assessment will be performed and a 'Preferred Reporting Items for Systematic Reviews and Meta-Analyses (PRISMA) flowchart', based on the PRISMA guidelines for the systematic review, will be created on the COVIDENCE platform.

\section{Selection process}

Initially, all duplicate articles will be checked by three reviewers (SB, NY and CTLP) individually through a double blinded process to assure a rigorous review approach and then appropriately rejected before identifying a unique set of articles for this study on the COVIDENCE platform.

Afterwards, the screening of title and abstracts of potential articles will be conducted by the same reviewers in a similar doubleblinded manner to assure rigour of the study. ${ }^{25}$ In case of any conflict related to the inclusion of an article, it will be temporarily included in the next step for further evaluation.

In the next step, full-text articles will be examined based on the eligibility criteria before final inclusion in the study. Reasons for the inclusion and exclusion of articles will be clearly identified and documented by each reviewer independently on online supplemental material B (Form for Screening of full text articles based on inclusion \& exclusion criteria with documented justification). Assuring three reviewers will allow for a consensus-based conflict resolution in case there is any uncertainty raised about inclusion of an article for the study. Moreover, the fourth researcher will also be consulted before finalising the final set of included articles for this study.

\section{Data collection process}

Data will be extracted based on online supplemental material C (Data Extraction Form for Included Studies) by three reviewers (SB, NY and CTLP) independently in a blind manner for each included study. SB will randomly select half of the included studies, which will be given to the fourth researcher to extract data independently. This will ensure rigour of the study and a consensus based approach for approving the final content of the data extraction form. Any disagreements will be resolved through mediation with the fourth researcher. The data extraction form will also offer 
content on the risk of bias and quality assessment of included studies. As the final data synthesis will involve using a conceptual framework, all relevant quantitative data will be converted into meaningful qualitative data for analysis and interpretation.

\section{Data items}

The descriptive data items that will be collected constitute: (1) characteristics of thestudyincluding country and year of publication, research aim and participant characteristics; (2) methodological characteristics including study design, data/ information collection methods and data analysis; and (3) results associated with the readiness of the public health system to tackle the burden of IHD along with concluding remarks from the article. The data collection items will also include risk of bias and quality assessment of included individual articles based on appropriate tools. The proposed tools and process for assessment of risk of bias and quality assessment of eligible studies are further detailed in the 'Risk of bias in individual studies' section within this protocol. For this study, we wish to create simplification of quantitative data by transforming them to meaningful qualitative units to accommodate them into the conceptual framework opted for our study. Moreover, only relevant data will be extracted based on the needs of the study.

We will extract data from those studies concerned with primary care facilities where facility assessment can provide data pertaining to facility readiness even if readiness concerning IHD is not mentioned.It is because the treatment of IHD and its risk factors are integrated at the primary care level along with treatment of other communicable diseases and NCDs. ${ }^{26}$

\section{Outcomes and prioritisation}

Outcomes of this study will constitute both quantitative and qualitative data retrieved from the included articles to inform health system factors that influence the readiness of India's public health system to tackle the burden of IHD.

This systematic review will include data at all levels of care that inform health service delivery, health workforce, health information system, access to essential medicines, health financing and health system governance concerning the present topic.

In terms of quantitative data, data from facility readiness assessment, health facility assessment, WHO Service Availability and Readiness Assessment data, surveys, baseline study of health facilities and monitoring and evaluation data of health facilities, along with data from the national NCD programme of India will serve to provide information on the outcome.

In terms of qualitative studies, data from in-depth interviews, focus group discussions, researcher observations of facilities and data from the NHPs will serve to understand the outcomes better.

\section{Risk of bias in individual studies}

All eligible studies at the outcome stage are expected to have methodological variation in approach, and therefore, we will assess their quality and risk of bias at this stage. In order to minimise the risk of bias and evaluate the quality of included studies, the Cochrane collaboration tool for assessing the risk of bias of randomised control trials, Newcastle-Ottawa Scale for cohort and casecontrol studies, NIH Quality Assessment Tool for CrossSectional Studies and Critical Appraisal Skills Programme tool for qualitative studies will be used based on the methodological design of the individual eligible study. ${ }^{27-30}$

We will not reject any study based on the assessed quality only; the information will be documented in the data extraction and synthesis stage. The risk of bias assessment and quality appraisal will be independently performed by each reviewer before converging to a mutually agreed decision. Any discrepancies will be mediated through the fourth researcher.

NHPs of India, national NCD programme guidelines and raw primary data from the OGD Platform cannot be assessed for risk of bias and quality appraisal. Therefore, they will be excluded from this process of evaluation.

\section{Data synthesis}

The extracted data will be analysed through a conceptual framework, the 'WHO Framework for Action', followed by summarisation of findings to answer the research questions. We do not expect to conduct a meta-analysis as we do not anticipate many articles in which meta-analysis may be appropriate. Additionally, the inclusion of qualitative studies will likely lead to a heterogeneous outcome which is not suitable for meta-analysis. Moreover, we will be transforming the quantitative data to meaningful qualitative units for assimilation into the conceptual framework for our study. Data analysis through the proposed conceptual framework rather serves as a conceptual lens for this study. Therefore, we will be deductive in our approach, assuming all health system readiness issues are inclusive within this conceptual framework. The conceptual framework was chosen through mediation with all reviewers, and moreover, straightforward demarcation of health system components allow for clear interpretation. ${ }^{31}$ We also considered other conceptual frameworks; however, they were either overly complex for the purposes of this study or considered factors beyond the health system that were not relevant for the present study. ${ }^{32-36}$

\section{Subgroup analysis}

We anticipate that subgroup analysis might be performed from the extracted data by using the components of 'health system strengthening' (HSS) building blocks from the proposed conceptual framework.

\section{Data summary}

Data will be summarised thematically based on HSS building blocks from the proposed conceptual framework. 


\section{Metabias(es)}

We do not plan to conduct any assessment of meta-bias (es) as the expected studies included for this systematic review might be of varied study design, making it difficult to assess such bias such as 'publication bias across studies' or 'selective reporting within studies'. ${ }^{37} 38$

In this context, our approach to this review might be influenced by 'language bias' as we are including studies published only in English. We plan to mention this aspect as our study limitation in the final review. Further, performing citation tracking can lead us to a 'biased sample of studies'.39 We plan to mitigate this by involving multiple authors in the screening, data extraction and data analysis process. Moreover, using a particular conceptual framework with a deductive research approach might lead us to a selective reporting of outcomes. We plan to avoid this through collective discussions between all the researchers post data extraction stage.

\section{Confidence in cumulative evidence}

At present, we do not have plans to assess the confidence in cumulative evidence as this study does not report effectiveness of clinical outcomes.

\section{Presenting or reporting the results}

The proposed systematic review will transparently represent the entire screening process and offer information on the finally included studies through a flow diagram in accordance with the Preferred Reporting Items for Systematic Review and Meta-Analysis Protocols guidelines. ${ }^{40}$ Data collection will be through a data extraction form with outcomes to extract data related to health system readiness. Data will be summarised in tables with details of study characteristics, methodological characteristics and individual study results. We will also provide data of included and excluded studies with documented justification for their inclusion or exclusion.

\section{Patient and public involvement}

The present study is a systematic review and not an empirical study. Therefore, there is no direct involvement of patients or the public in this study.

\section{Ethics and dissemination \\ Ethical issues}

This study, being a systematic review, does not involve any clinical trial, primary data collection or empirical study involving humans or animals. Therefore, no ethical permissions were sought by the reviewers.

\section{Publication plan}

We plan to publish the preliminary results of this review in conferences and to proceed on to publish the final article in a peer-reviewed journal once completed. We hope to publish both the protocol and systematic review in open access journals to benefit academic communities, policymakers and other public health stakeholders with the ambition to improve the health system readiness of India to tackle the burden of IHD.
Twitter Shuvarthi Bhattacharjee @ShuvarthiB, Nima Yaghmaei @NimaYaghmaei and Dinesh Neupane @Dines2021

Contributors SB is the guarantor of the review. SB and NY conceptualised the study. A search strategy will be created and run on relevant platforms by SB. All search results will be retrieved by SB from appropriate databases, trial registry (for details of primary studies in reviews) and grey literature. CTLP an NY will validate the search strategy prior to implementation and check the retrieved search results. $\mathrm{SB}, \mathrm{NY}$ and CTLP will screen articles, and any discrepancies for final inclusion of full- text articles will be resolved by DN (fourth researcher). All authors will be involved in data extraction, quality appraisal, data synthesis and formulation of the final draft.

Funding The authors have not declared a specific grant for this research from any funding agency in the public, commercial or not-for-profit sectors.

Competing interests None declared.

Patient and public involvement statement The present study is a systematic review and not an empirical study. Therefore, there is no direct involvement of patients or the public in this study.

Patient consent for publication Not required.

Ethics approval Not required. As this is a review only.

Provenance and peer review Not commissioned; externally peer reviewed.

Supplemental material This content has been supplied by the author(s). It has not been vetted by BMJ Publishing Group Limited (BMJ) and may not have been peer-reviewed. Any opinions or recommendations discussed are solely those of the author(s) and are not endorsed by BMJ. BMJ disclaims all liability and responsibility arising from any reliance placed on the content. Where the content includes any translated material, BMJ does not warrant the accuracy and reliability of the translations (including but not limited to local regulations, clinical guidelines, terminology, drug names and drug dosages), and is not responsible for any error and/or omissions arising from translation and adaptation or otherwise.

Open access This is an open access article distributed in accordance with the Creative Commons Attribution Non Commercial (CC BY-NC 4.0) license, which permits others to distribute, remix, adapt, build upon this work non-commercially, and license their derivative works on different terms, provided the original work is properly cited, appropriate credit is given, any changes made indicated, and the use is non-commercial. See: http://creativecommons.org/licenses/by-nc/4.0/.

\section{ORCID iDs}

Shuvarthi Bhattacharjee http://orcid.org/0000-0002-6079-5365

Nima Yaghmaei http://orcid.org/0000-0001-7740-8037

Cao Tran Le Phuong http://orcid.org/0000-0003-3538-6604

Dinesh Neupane http://orcid.org/0000-0002-1501-2990

\section{REFERENCES}

1 Institute for Health Metrics and Evaluation (IHME). Findings from the global burden of disease study 2019. Seattle, WA: IHME, 2021. https://vizhub.healthdata.org/gbd-compare/

2 World Health Organization (WHO). Global health expenditure database, 2020. Available: http://www.who.int/health-accounts/ ghed/en/

3 Ministry of Health \& Family Welfare (MoHFW, India). National health portal (2019, November 5). Non-communicable diseases, 2019. Available: https://www.nhp.gov.in/healthlyliving/ncd2019

4 WHO. Global action plan for the prevention and control of noncommunicable diseases 2013-2020. Geneva: World Health Organization, 2013: 102.

5 Ministry of Health \& Family Welfare (MoHFW, India). National action plan and monitoring framework for prevention \& control of noncommunicable diseases (NCDs), 2020. Available: https://www. iccp-portal.org/system/files/plans/India\%20-\%20National_Action_ Plan_and_Monitoring_Framework_Prevention_NCD_2013.pdf

6 Ministry of Health \& Family Welfare (MoHFW, India). National health policy. India Ministry of Health \& Family Welfare, Government of India.; 2017: 1-28. https://www.nhp.gov.in/nhpfiles/national_health_ policy_2017.pdf [Accessed 04.08.2021].

7 Ministry of Health \& Family Welfare (MoHFW, India), Directorate General of Health Services (DGHS). National programme for prevention and control of cancer, diabetes, cardiovascular diseases and stroke, 2020. Available: https://dghs.gov.in/content/1363_3_Nati onalProgrammePreventionControl.aspx 
8 Gopichandran V. Ayushman Bharat National health protection scheme: an ethical analysis. Asian Bioeth Rev 2019;11:69-80.

9 World Health Organization. Tackling NCDs: 'best buys' and other recommended interventions for the prevention and control of noncommunicable diseases. Geneva: World Health Organization, 2017.

10 Narain JP. Public health challenges in India: seizing the opportunities. Indian J Community Med 2016;41:85.

11 Tiwari R, Negandhi H, Zodpey SP. Health management workforce for India in 2030. Front Public Health 2018;6:227.

12 Lakshminarayanan S. Role of government in public health: current scenario in India and future scope. J Family Community Med 2011;18:26.

13 Abazinab S, Woldie M, Alaro T. Readiness of health centers and primary hospitals for the implementation of proposed health insurance schemes in Southwest Ethiopia. Ethiop J Health Sci 2016;26:449.

14 Marin TS, Walsh S, May N, et al. Screening for depression and anxiety among patients with acute coronary syndrome in acute care settings: a scoping review. JBI Evid Synth 2020;18:1932-69.

15 Likka MH, Handalo DM, Weldsilase YA, et al. The effect of community-based health insurance schemes on utilization of healthcare services in low- and middle-income countries: a systematic review protocol of quantitative evidence. JBI Database System Rev Implement Rep 2018;16:653-61.

16 Teferra MN, Ramos JS, Kourbelis C, et al. Electronic textile-based electrocardiogram monitoring in cardiac patients: a scoping review. JBI Database System Rev Implement Rep 2019;17:1958-98.

17 Ninan A, Acosta J, Kulesza T, et al. A systematic review of the effectiveness of preventive measures for coronary artery disease in Asian Indians. JBI Libr Syst Rev 2011;9:1-17.

18 Ninan A, Acosta J, Kulesza T, et al. A systematic review on the risk factors of coronary artery disease found in Asian Indians. JBI Libr Syst Rev 2011;9:1-14.

19 Kumar P, Kumar M. Management of potential ventilator shortage in India in view of on-going COVID-19 pandemic. Indian J Anaesth 2020;64:151.

20 lyengar KP, Jain VK, Vaishya R. Current situation with doctors and healthcare workers during COVID-19 pandemic in India. Postgraduate medical Journal 2020. doi:10.1136/ postgradmedj-2020-138496

21 World Health Organization. Everybody's business-strengthening health systems to improve health outcomes: WHO's framework for action. Geneva World Health Organization (WHO); 2007: 1-44. https://apps.who.int/iris/bitstream/handle/10665/43918/ 9789241596077_eng.pdf [Accessed 04.08.2021]. 9789241596077.

22 Arokiasamy P. India's escalating burden of non-communicable diseases. Lancet Glob Health 2018;6:e1262-3.

23 Wright K, Golder S, Rodriguez-Lopez R. Citation searching: a systematic review case study of multiple risk behaviour interventions. BMC Med Res Methodol 2014;14:73.

24 covidence. Covidence systematic review software, veritas health innovation, Melbourne, Australia, 2021. Available: www.covidence. org
25 Ayala Quintanilla BP, Taft A, McDonald S, et al. Social determinants and maternal exposure to intimate partner violence of obstetric patients with severe maternal morbidity in the intensive care unit: a systematic review protocol. BMJ Open 2016;6:e013270.

26 Ministry of Health \& Family Welfare, Government of India. National programme for prevention and control of cancer, diabetes, cardiovascular disease and stroke: operational guidelines(Revised 2013-17). India Directorate General of Health Services Ministry of Health \& Family welfare. Government Of India.; 2013: 1-78. https:// main.mohfw.gov.in/sites/default/files/Operational\%20Guidelines\% 20of\%20NPCDCS\%20\%28Revised\%20-\%202013-17\%29_1.pdf [Accessed 04.08.2021].

27 Higgins J, Altman D, Gotzsche P. The Cochrane Collaboration's tool for assessing risk of bias in randomised trials. BMJ 2011;343:d5928-d5928.

28 Peterson J, Welch V, Losos M, et al. The Newcastle-Ottawa scale (NOS) for assessing the quality of nonrandomised studies in metaanalyses. Ottawa: Ottawa Hospital Research Institute, 2011.

29 National Institutes of Health (NIH). Quality assessment tool for observational cohort and cross-sectional studies. 2014. available online at. Available: https://www.nhlbi.nih.gov/health-topics/studyquality-assessment-tools [Accessed November 19, 2020].

30 Critical Appraisal Skills Programme. Casp) qualitative checklist 2018. Available: https://casp-uk.net/wp-content/uploads/2018/01/ CASP-Qualitative-Checklist-2018.pdf [Accessed 18 Sept. 2020].

31 Mounier-Jack S, Griffiths UK, Closser S, et al. Measuring the health systems impact of disease control programmes: a critical reflection on the who building blocks framework. BMC Public Health 2014;14:278.

32 Roberts MJ, Hsiao WC, Berman P, et al. Getting health reform right. 27. New York: Oxford University Press, page, 2003.

33 De Savigny D, Adam T, eds. Systems thinking for health systems strengthening. Geneva: World Health Organization, 2009.

34 Shakarishvili G, Lansang MA, Mitta V, et al. Health systems strengthening: a common classification and framework for investment analysis. Health Policy Plan 2011;26:316-26.

35 Olmen Jvan, Criel B, Damme WV, et al. Analysing health systems to make them stronger.ITG press. Belgium: Antwerpen, 2010.

36 Murray CJ, Frenk J. A framework for assessing the performance of health systems. Bull World Health Organ 2000;78:717-31.

37 Dalton JE, Bolen SD, Mascha EJ. Publication bias. Anesthesia \& Analgesia 2016;123:812-3.

38 Reid EK, Tejani AM, Huan LN, et al. Managing the incidence of selective reporting bias: a survey of cochrane review groups. Syst Rev 2015;4:85.

39 Higgins JPT, Thomas J, Chandler J, et al, eds. Cochrane Handbook for systematic reviews of interventions version 6.2 (updated February 2021). London: Cochrane, 2021. www.training.cochrane.org/ handbook

40 Moher D, Liberati A, Tetzlaff J, et al. Preferred reporting items for systematic reviews and meta-analyses: the PRISMA statement. PLoS Med 2009;6:e1000097. 